\title{
VLBA determinations of the distances to nearby star-forming regions
}

\author{
L. Loinard ${ }^{1}$, R. M. Torres ${ }^{1}$, \\ A. J. Mioduszewski ${ }^{2}$ and L.F. Rodríguez ${ }^{1}$ \\ ${ }^{1}$ Centro de Radiastronomía y Astrofísica, Universidad Nacional Autónoma de México \\ Apartado Postal 72-3 (Xangari), 58089 Morelia, Michoacán, México \\ email: 1.1oinard,r.torres, 1.rodriguez@astrosmo.unam.mx \\ ${ }^{2}$ National Radio Astronomy Observatory, Array Operations Center \\ 1003 Lopezville Road, Socorro, NM 87801, USA \\ email: amiodusz@aoc.nrao.edu
}

\begin{abstract}
Using phase-referenced multi-epoch Very Long Baseline Array observations, we have measured the trigonometric parallax of several young stars in the Taurus and Ophiuchus starforming regions with unprecedented accuracy. The mean distance to the Taurus complex was found to be about $140 \mathrm{pc}$, and its depth around $20 \mathrm{pc}$, comparable to the linear extent of Taurus on the sky. In Ophiuchus, 4 sources have been observed so far. Two of them were found to be at about $160 \mathrm{pc}$ (the distance traditionally attributed to Ophiuchus), while the other 2 are at about 120 pc. Since the entire Ophiuchus complex is only a few parsecs across, this difference is unlikely to reflect the depth of the region. Instead, we argue that two physically unrelated sites of star-formation are located along the line of sight toward Ophiuchus.
\end{abstract}

Keywords. astrometry, stars: distances, stars: formation, radio continuum: stars, stars: magnetic fields, radiation mechanisms: nonthermal, techniques: interferometric, binaries: general, ISM: clouds

\section{Introduction}

To provide accurate observational constraints for pre-main sequence evolutionary models, and thereby improve our understanding of star-formation, it is crucial to measure as accurately as possible the properties (e.g., age, mass, luminosity) of individual young stars. The determination of most of these parameters, however, depends critically on the often poorly-known distance to the object. While the average distance to nearby low-mass star-forming regions (e.g. Taurus or $\rho$ Oph) has been estimated to about $20 \%$ precision using indirect methods (Elias 1978a,b; Kenyon et al. 1994; Knude \& Hog 1998; Bertout \& Genova 2006), the line-of-sight depth of these regions is largely unknown, and accurate distances to individual objects are still missing. Even the highly successful Hipparcos mission (Perryman et al. 1997) did little to improve the situation (Bertout et al. 1999) because young stars are heavily embedded in their parental clouds and are, therefore, faint in the optical band observed by Hipparcos.

Low-mass young stars often generate non-thermal continuum emission produced by the interaction of free electrons with the intense magnetic fields that tend to exist near their surfaces (e.g. Feigelson \& Montmerle 1999). If the magnetic field intensity and the electron energy are sufficient, the resulting compact radio emission can be detected with Very Long Baseline Interferometers (VLBI - e.g. André et al. 1992). The relatively recent possibility of accurately calibrating the phase of VLBI observations of faint, compact radio sources using nearby quasars makes it possible to measure the absolute position of these objects (or, more precisely, the angular distance between them and the calibrating 
Table 1. Distances to the sources in Taurus and Ophiuchus

Unit: pc

\begin{tabular}{lccccccc}
\hline Complex & \multicolumn{3}{c}{ Taurus } & \multicolumn{5}{c}{ Ophiuchus } \\
\hline Source & T Tau & Hubble 4 & HDE 283572 & S1 & DoAr 21 & VSSG 14 & WL 5 \\
Distance & $147.6 \pm 0.6$ & $132.8 \pm 0.5$ & $128.5 \pm 0.6$ & $116.9_{-6.4}^{+7.2}$ & $121.9_{-5.3}^{+5.8}$ & $165.6_{-5.8}^{+6.2}$ & $168.3_{-9.3}^{+8.2}$ \\
\hline
\end{tabular}

quasar) to better than a tenth of a milli-arcsecond (Brisken et al. 2000, 2002; Loinard et al. 2005, 2007; Torres et al. 2007; Xu et al. 2006; Hachisuka et al. 2006; Hirota et al. 2007; Sandstrom et al. 2007). This level of precision is sufficient to constrain the trigonometric parallax of sources within a few hundred parsecs of the Sun (in particular of nearby young stars) with a precision better than a few percent using multi-epoch VLBI observations. With this goal in mind, we have initiated a large project aimed at accurately measuring the trigonometric parallax of a sample of magnetically active young stars in the most prominent and often-studied northern star-forming regions within 1 kpc of the Sun (Taurus, $\rho$-Ophiuchus, Perseus, Serpens). We use the 10-element Very Long Baseline Array (VLBA) of the National Radio Astronomy Observatory (NRAO). Here, we will summarize the results obtained so far in Taurus and Ophiuchus.

\section{Observations and parallax determination}

All the observations used here were obtained in the continuum at $3.6 \mathrm{~cm}(8.42 \mathrm{GHz})$ with the VLBA of the National Radio Astronomy Observatory (NRAO). A total of 7 sources were studied so far: three in Taurus (T Tau, Hubble 4, and HDE 283572), and four in Ophiuchus (S1, DoAr 21, VSSG 14, and WL 5). In all cases, between 6 and 12 observations spread over 1.5 to 2 years were obtained. The data were edited and calibrated following the standard VLBA procedures for phase-referenced observations (see Loinard et al. 2007, and Torres et al. 2007 for details).

The displacement of the sources on the celestial sphere is the combination of their trigonometric parallax $(\pi)$ and proper motion $(\mu)$. For isolated sources (such as Hubble 4, and HDE 283572 in our case), it is common to consider linear and uniform proper motions, so the right ascension and declination vary as a function of time $t$ as:

$$
\begin{aligned}
\alpha(t) & =\alpha_{0}+\left(\mu_{\alpha} \cos \delta\right) t+\pi f_{\alpha}(t) \\
\delta(t) & =\delta_{0}+\mu_{\delta} t+\pi f_{\delta}(t),
\end{aligned}
$$

where $\alpha_{0}$ and $\delta_{0}$ are the coordinates of the source at a given reference epoch, $\mu_{\alpha}$ and $\mu_{\delta}$ are the components of proper motion, and $f_{\alpha}$ and $f_{\delta}$ are the projections over $\alpha$ and $\delta$, respectively, of the parallactic ellipse.

For sources in multiple systems, however, the proper motions are affected by the gravitational influence of the other members of the system. As a consequence, the motions are curved and accelerated, rather than linear and uniform. If the orbital period is long compared with the timespan covered by the observations (as it is for T Tau -see Loinard et al. 2003), it is sufficient to include a uniform acceleration in the fit. This leads to the functions:

$$
\alpha(t)=\alpha_{0}+\left(\mu_{\alpha 0} \cos \delta\right) t+\frac{1}{2}\left(a_{\alpha} \cos \delta\right) t^{2}+\pi f_{\alpha}(t)
$$



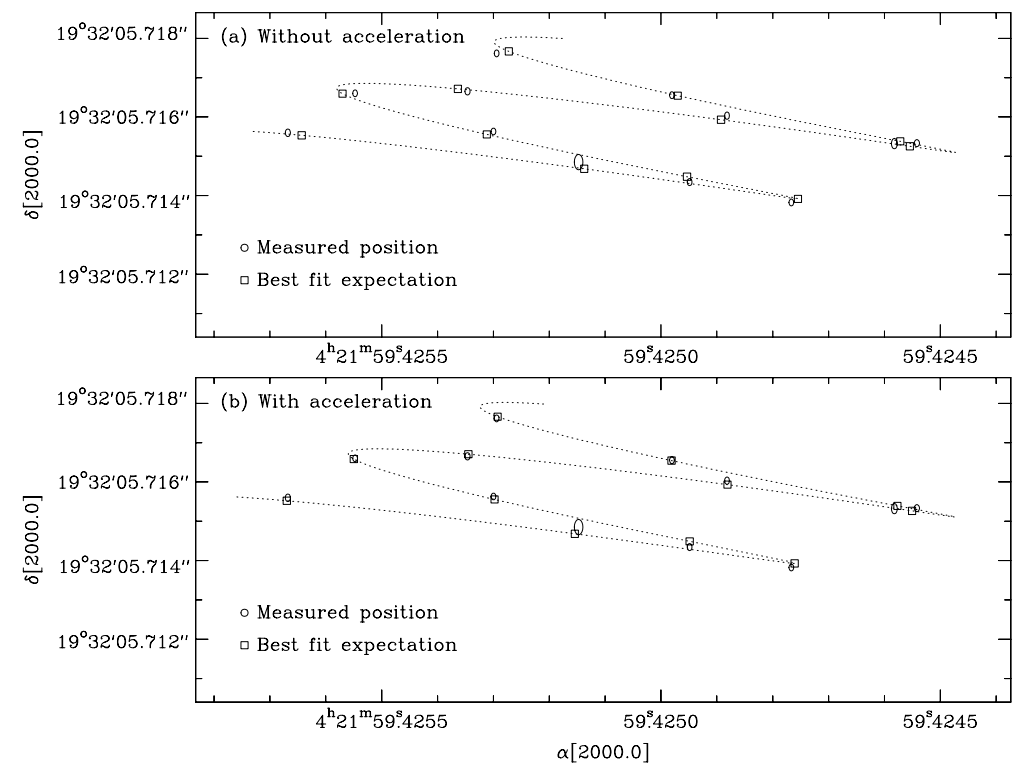

Figure 1. Measured positions of $\mathrm{T} \mathrm{Tau} \mathrm{Sb}$ and best fit without (a) and with (b) acceleration terms. The observed positions are shown as ellipses, the size of which represents the magnitude of the errors. Note the very significant improvement when acceleration terms are included.

$$
\delta(t)=\delta_{0}+\mu_{\delta 0} t+\frac{1}{2} a_{\delta} t^{2}+\pi f_{\delta}(t),
$$

where $\mu_{\alpha 0}$ and $\mu_{\delta 0}$ are the proper motions at a reference epoch, and $a_{\alpha}$ and $a_{\delta}$ are the projections of the uniform acceleration (see Fig. 1 for a comparison between fits with and without acceleration terms).

Finally, if a source is a member of a multiple system whose orbital period is shorter than, or comparable with the timespan covered by the observations (as will be the case for all Ophiuchus sources), then a full Keplerian fit is needed, but additional observations are required to properly constrain that fit. These observations are currently being obtained for all Ophiuchus sources, but are not yet analyzed. As a consequence, the fits presented below for the Ophiuchus sources are based on Eqs. 2.1 and 2.2. The resulting uncertainties will be much larger than for the sources in Taurus because the orbital motions generate an unmodelled scatter around the mean positions of sources. These increased errors should disappear once we include new observations, and perform a full Keplerian fit.

\section{Results and discussion}

The distance to the seven sources studied here are given in Table 1. For the Taurus sources, Hipparcos parallaxes (Bertout et al. 1999), and/or estimates based on a modified convergent point method (Bertout \& Genova 2006) are available. Our measurements are always consistent with these values, but ours are one to two orders of magnitude more accurate. Only one source in Taurus (V773 Tau; Lestrade et al. 1999) has a VLBI-based parallax determination. Taking the mean of that and our 3 measurements, we estimate the mean distance to the Taurus cluster to be $\bar{d}=137$ pc. The dispersion about that mean leads to a full width at half maximum depth of about $20 \mathrm{pc}$, comparable to the linear extent of Taurus on the sky.

Traditionally assumed to be at a distance of 165 pc (Chini 1981), Ophiuchus has recently been proposed to be somewhat nearer, at 120 pc (Knude \& Hog 1998). Interest- 
ingly, two of our sources are consistent with the traditional value, but the other two are consistent with the distance at 120 pc. Since Ophiuchus is only a few parsecs across on the plane of the sky, it is very unlikely to have a depth of $40 \mathrm{pc}$. It is noteworthy that the two sources at about 120 pc (S1 and DoAr 21) are associated with the sub-condensation Oph A, whereas the two sources at $\sim 165$ pc (VSSG 14 and WL 5) are associated with the condensation Oph B (See Motte et al. 1998 for an overview of the Ophiuchus complex). Thus, a plausible explanation of our results is that two physically unrelated star-forming regions are located along the line-of-sight toward Ophiuchus. Interestingly, Knude \& Hog (1998) noticed an effect that would be consistent with this possibility in their analysis of extinction towards Ophiuchus. While a clear extinction step was visible at 120 pc, extinction was apparently extending up to about $160 \mathrm{pc}$. Additional VLBI observations will be needed to confirm the existence of two regions of star-formation towards Ophiuchus, and to investigate at which distance the other condensations in Ophiuchus are located.

\section{Conclusions and perspectives}

The present results show that VLBA observations of non-thermal sources associated with young stars have the potential to improve very significantly our knowledge of the spatial distribution for star-forming regions in the Solar neighborhood. Indeed, the precision obtained by these measurements is sufficient even to probe a 3D structure of star-forming regions. Coupled with pre-main sequence evolutionary models, such information could be used to reconstruct the history of star-formation in individual regions.

\section{References}

Andre, P., Deeney, B. D., Phillips, R. B., \& Lestrade, J.-F. 1992, ApJ, 401, 667

Bertout, C., Robichon, N., \& Arenou, F., 1999, A\&A, 352, 574

Bertout, C. \& Genova, F. 2006, A\&A, 460, 499

Brisken, W. F., Benson, J. M., Beasley, A. J., Fomalont, E. B., Goss, W. M., \& Thorsett, S. E. 2000, ApJ, 541, 959

Brisken, W. F., Benson, J. M., Goss, W. M., \& Thorsett, S. E. 2002, ApJ, 571, 906

Chini, R. 1981, A\&A, 99, 346

Elias, J. H. 1978a, ApJ, 224, 857

Elias, J. H. 1978b, ApJ, 224, 453

Feigelson, E. D. \& Montmerle, T., 1999, ARAA, 37, 363

Hachisuka, K., et al. 2006, ApJ, 645, 337

Hirota, T., et al. PASJ, in press (http://arxiv.org/abs/0705.3792)

Kenyon, S. J., Dobrzycka, D., \& Hartmann L., 1994, AJ, 108, 1872

Knude, J. \& Hog, E. 1998, A\&A, 338, 897

Lestrade, J.-F., Preston, R. A., Jones, D. L., et al., 1999, A\&A, 344, 1014

Loinard, L., Torres, R. M., Mioduszewski, A. J., Rodríguez, L. F., González, R. A., Lachaume, R., Vázquez, V., \& González, E. 2007, ApJ, 671, 546

Loinard, L., Mioduszewski, A. J., Rodríguez, L. F., González, R. A., Rodríguez, M. I., \& Torres, R. M. 2005, ApJL, 619, L179

Loinard L., Rodríguez, L. F., \& Rodríguez, M. I., 2003, ApJ, 587, L47

Motte, F., Andre, P., \& Neri, R. 1998, A\&A, 336, 150

Perryman, M. A. C., Lindegren, L., Kovalevsky, J., et al., 1997, A\&A, 323, L49

Sandstrom K. M., et al., ApJ, in press (http://arxiv.org/abs/0706.2361)

Torres, R. M., Loinard, L., Mioduszewski, A. J., \& Rodríguez, L. F. 2007, ApJ, in press

Xu, Y., Reid, M. J., Zheng, X. W., \& Menten, K. M. 2006, Science, 311, 54 